\title{
Risk factors for early neonatal sepsis in the term baby
}

\author{
*K S Y Perera ${ }^{1}$, M Weerasekera ${ }^{2}$, U D T M Weerasinghe ${ }^{3}$
}

Sri Lanka Journal of Child Health, 2018; 47(1): 44-49

\begin{abstract}
Introduction: Neonatal sepsis is a clinical syndrome with systemic signs and symptoms of infection within the first 4 weeks of life.
\end{abstract}

Objectives: To determine the incidence of perinatal sepsis in term babies born at Sri Jayewardenepura General Hospital (SJGH), to evaluate the disease pattern of term sick neonates born at SJGH and to estimate and compare the incidence of maternal risk factors, individually and in combination with the control group.

Method: A retrospective case control study was carried out from September 2014 to August 2015 on all term babies born at SJGH showing clinical and biochemical evidence of sepsis. The information was collected from the clinical records. The Chi Square test was used to assess significant associations. The level of significance was 0.05 .

Results: Out of 3,482 term deliveries 161 (4.6\%) showed biochemical and/or microbiological evidence of sepsis. The control group consisted of 120 term neonates. Septicaemia was diagnosed in 6 $(0.2 \%)$ term neonates. Congenital pneumonia was diagnosed in $15(0.45 \%)$ neonates. Twenty five $(15 \%)$ mothers of the sick neonates had prolonged rupture of membranes (PROM) lasting 18 hours and more. Fifty seven (35\%) of the mothers had a labour room stay of 9 hours or more. One hundred and eighteen $(73 \%)$ mothers had 3 or more vaginal examinations. Thirty four (21.1\%) mothers underwent induction of labour. Six $(3.7 \%)$ had maternal fever. Three (1.8\%) had foul smelling liquor and $2(1.2 \%)$ had both. Two (1.2\%) had high vaginal swab (HVS) positive for Group B Streptococcus (GBS).

${ }^{1}$ Medical officer, ${ }^{2}$ Consultant Neonatologist,
Neonatal Intensive Care Unit, ${ }^{3}$ Medical Officer,
Reproductive Health, Sri Jayewardenepura
General Hospital, Sri Lanka
*Correspondence: moonstone12345@hotmail.com

(Received on 12 May 2017: Accepted after revision on 16 June 2017)

The authors declare that there are no conflicts of interest

Personal funding was used for the project.

Open Access Article published under the Creative

Commons Attribution CC-BY (CC) (P)
Conclusions: Neonates with biochemical evidence of sepsis showed a statistically significant incidence of PROM lasting 18 hours or more, labour room stay more than 9 hours and 3 or more vaginal examinations. There was no significant difference in occurrence of labour induction, maternal fever, HVS positivity for GBS and foul smelling liquor.

DOI: http://dx.doi.org/10.4038/sljch.v47i1.8429

(Keywords: Sepsis, prolonged rupture of membranes, induction of labour, Group B Streptococcus, maternal fever)

\section{Introduction}

Neonatal sepsis is defined as a clinical syndrome with systemic signs and symptoms of infection within the first 4 weeks of life ${ }^{1}$. Pathogenic bacteria can gain access to the blood stream, causing overwhelming infection without much localization (septicaemia) or may be predominantly localized to the lungs (pneumonia) or the meninges (meningitis) or the urinary tract ${ }^{1}$. Early onset sepsis is defined as sepsis presenting within 72 hours of birth $^{2}$. According to neonatal deaths reported to the Registrar General's Office, Sri Lanka, 17,946 neonatal deaths have occurred from 1997 to 2001. Around $90 \%$ of deaths were during the first week of life and $19.8 \%$ were due to infection ${ }^{3}$. Sepsis commonly presents with nonspecific symptoms ranging from feeding difficulties, fever or hypothermia, to dyspnoea, grunting, cyanosis and apnoea ${ }^{4}$.

According to the NICE clinical guidelines, UK, the most serious risk factors for neonatal sepsis are confirmed or suspected invasive bacterial infection in the mother at any time during labour or within 24 hours after delivery, necessitating parenteral antibiotics and suspected or confirmed infection in one baby of a multiple pregnancy. The presence of either of these risk factors requires prophylactic antibiotics for the neonate. A combination of any two or more of the following risk factors also requires prophylactic antibiotics for the neonate:

1. Invasive group B streptococcal infection in a previous baby

2. Maternal group B streptococcal colonisation in the current pregnancy

3. Pre-labour rupture of membranes. (Rupture of membranes prior to the onset of labour). 
4. Preterm birth following spontaneous labour (before 37 weeks gestation)

5. Suspected or confirmed rupture of membranes for 18 hours or more in a preterm birth

6. Intra-partum fever higher than $38^{\circ} \mathrm{C}$ or confirmed or suspected chorioamnionitis ${ }^{2}$

Seven or more vaginal examinations during labour have also been found to increase the risk of infection (mothers or their babies) ${ }^{2}$. Active labour exceeding 9 hours, increases the incidence of perinatal sepsis 5 .

The National Guidelines for Newborn Care 2014, Sri Lanka, states that "prophylactic antibiotics should be considered in the following circumstances which are risk factors for early onset sepsis"

1. Foul smelling liquor or malodorous baby

2. When 2 or more of the following risk factors are present:

- Maternal pyrexia $>38^{\circ} \mathrm{C}$ or other evidence of infection

- Prolonged rupture of membranes (PROM) 18 or more hours

- Fetal distress (tachycardia, bradycardia, abnormal CTG)

- Passage of meconium in-utero

- $\quad$ Spontaneous preterm delivery $(<37$ weeks)

- Low Apgar $<7$ at 5 minutes

- Prolonged or difficult delivery with instrumentation or $\geq 3$ vaginal examinations or presence / removal of cervical suture

- Maternal UTI in the third trimester

3. Unclean delivery and cord separation

4. Previous baby affected with Group B Streptococcus (GBS) and mother's recent GBS status unknown or inadequately treated ${ }^{4}$.

When multiple risk factors are associated, the risk of neonatal sepsis increases significantly. Premature rupture of membranes for more than 24 hours before delivery without other complications increases the incidence of neonatal sepsis by $1 \%$. When combined with chorioamnionitis the incidence of neonatal infection is quadrupled ${ }^{6}$. The clinical signs of sepsis are non-specific and are also observed with other non-infectious conditions, making it difficult to clinically confirm. Available diagnostic testing (abnormal neutrophil indices, low platelet counts, acute-phase reactants such as C-reactive protein (CRP), serum procalcitonin level and blood culture), are helpful in the presence of clinical criteria especially when the baby responds to antibiotic therapy. Urine culture, gastric aspirates, body surface cultures, tracheal aspirates, lumbar punctures are less commonly used ${ }^{7}$.

\section{Objectives}

1. To determine the incidence of perinatal sepsis in term babies born at Sri Jayewardenepura General Hospital (SJGH).

2. To evaluate the disease pattern of term sick neonates born at SJGH.

3. To estimate and compare the incidence of maternal risk factors, individually and in combination between sick \& healthy neonates

\section{Method}

A retrospective case control study was carried out at SJGH over a 12 month period from September 2014 to end of August 2015. The study group included all the term babies born at SJGH who showed clinical, biochemical evidence of sepsis. Exclusion criteria included all babies born outside and admitted to SJGH. The control group consisted of randomly chosen term babies born at SJGH with no signs of sepsis during the same time period. The sample size was calculated by OpenEpi.

Data Collection: The information was collected from the Clinical Records (BHTs). Term babies admitted due to symptoms/signs of sepsis and positive biochemical criteria were noted. Documented risk factors as per local guidelines were considered, individually as well as in combination. The risk factors considered were:

- $\quad$ Prolonged rupture of membranes (PROM)

- Maternal fever

- Evidence of chorioamnionitis

- Induction of labour with Foley catheter

- Prolonged labour room stay (9 hours or more)

- Number of vaginal examinations (3 or more)

- Blood cultures, ear swabs and serial CRPs were taken from all babies included in the study. The control group consisted of term babies who did not have positive biochemical or clinical criteria.

Ethical Issues: Personal and clinical details of the patients collected during the study are kept confidential. Approval was obtained from the Ethics Review Committee of SJGH. There are no conflicts of interest.

Statistical analysis: Data analysis was done using the statistics programme SPSS and "N-1" Chisquared test on Medcalc statistical software. The mean birth weight, the frequencies of the risk factors under analysis and the statistical significance between the incidence of risk factors in the septic and control groups were calculated. 


\section{Results}

The mean birth weight of the neonates included in the study was $3.21 \mathrm{~kg}$ and the commonest mode of delivery $(41.6 \%)$ was normal vaginal delivery. The mean birth weight of the control group was $3.05 \mathrm{~kg}$ and the commonest mode of delivery $(56.1 \%)$ was normal vaginal delivery. The total number of term deliveries during the study period was 3,482 out of which $161 \quad(4.6 \%)$ term neonates showed biochemical and/or microbiological evidence of sepsis. One hundred and twenty (3.5\%) term neonates were included in the control group.

Septicaemia (positive blood cultures) was diagnosed in $6(0.2 \%)$ term neonates. Out of the septicaemia group $83 \%$ were caused by GBS. Septicaemia with congenital pneumonia was diagnosed in $4(0.1 \%)$. Congenital pneumonia was diagnosed in $15(0.5 \%)$ neonates out of which 3 $(20 \%)$ were due to GBS infection. Meningitis was diagnosed in 3 (0.86 per 1000 live births). In the current study there were no deaths among the term neonates with early onset sepsis.

For the neonates who had symptoms/signs of sepsis the average time spent in the labour room was 6 hours. The average length of time of rupture of membranes was 8.6 hours and the average number of vaginal examinations was 4 . In the control group the average time spent in the labour room was 4 hours. The average length of time of rupture of membranes was 4 hours and the average number of vaginal examinations was 3 .

Twenty five mothers of sick, term neonates had PROM lasting 18 hours and more. This gave an incidence of $15 \%$. None of the mothers in the control group had PROM lasting 18 hours or more. Fifty seven mothers of sick babies had a labour room stay of 9 hours or more, which gives an incidence of $35 \%$. Fifteen mothers in the control group had a labour room stay of 9 hours or more with an incidence of $12.5 \%$. One hundred and eighteen mothers had 3 or more vaginal examinations, giving an incidence of $73 \%$. The control group had 51 mothers with 3 or more vaginal examinations. This gave an incidence of $42.5 \%$.

Thirty four $(21.1 \%)$ mothers underwent induction of labour. The most frequently used method of induction was Foley catheter induction, which included $23(14.3 \%)$. Induction of labour by prostaglandin was done in $11(6.8 \%)$ of mothers. Twenty $(16.6 \%)$ mothers in the control group underwent induction of labour. Ten mothers had Foley catheter induction and 10 had induction of labour by prostaglandin which gave an incidence of $8.3 \%$.
Six $(3.7 \%)$ mothers had maternal fever. Three $(1.8 \%)$ mothers had foul smelling liquor and 2 $(1.2 \%)$ had both. Two mothers (1.2\%) had high vaginal swab (HVS) positive for GBS. In the control group $2(1.7 \%)$ mothers had maternal fever and none had foul smelling liquor or HVS positive for GBS. Eighty mothers had two or more risk factors, leading to an incidence of 50\%. Forty two mothers had only one risk factor, giving an incidence of $26 \%$. Thirty nine mothers of sick, term neonates had no risk factors. This gives an incidence of $24 \%$. The control group had 19 $(15.8 \%)$ mothers with 2 or more risk factors. Thirty nine $(32.5 \%)$ mothers had only one risk factor. Sixty one $(50.8 \%)$ mothers had no risk factors.

In our study, all cases of septicaemia had 3 or more vaginal examinations. Twenty five percent of mothers of babies with GBS septicaemia had PROM lasting 18 hours or more. Seventy five percent of mothers with babies having GBS septicaemia had 9 or more hours in the labour room. Fifty percent of babies with GBS septicaemia had mothers whose induction of labour was by Foley catheter. None of the mothers had chorioamnionitis. The commonest cause $(66 \%)$ of septicaemia was GBS. The rest were Staphylococcus aureus. The current study found that maternal risk factors had a statistically significant effect on the incidence of sepsis with biochemical evidence in neonates. The incidence of ill health in term babies was $0.2 \%$. The commonest diagnosis was superficial sepsis $(2.9 \%)$. Fifty per cent of mothers had 2 or more risk factors. The commonest risk factor $(73 \%)$ was found to be 3 or more vaginal examinations. The second most frequently found risk factor (35\%) was 09 or more hours spent in the labour room.

In our study a statistically significant higher number of neonates with biochemical evidence of sepsis had PROM of 18 or more hours, labour room stay more than 9 hours and 3 or more vaginal examinations when compared to the control group $(p=<0.0001,95 \% \mathrm{CI}=9.0417$ to $21.4687, p=$ $<0.0001,95 \% \mathrm{CI}=12.1647$ to $32.0328, p=$ $<0.0001, \quad 95 \% \quad \mathrm{CI}=18.4781$ to 41.6906 ) respectively. There was no significant difference in occurrence of labour induction ( $p=0.3441,95 \%$ $\mathrm{CI}=-5.4235$ to 13.9195$)$, maternal fever $(p=$ $0.3085,95 \%$ CI $=-2.7842$ to 6.4856$)$, HVS positivity for GBS $(p=0.1403,95 \% \mathrm{CI}=-1.5529$ to 5.2558) and foul smelling liquor ( $p=0.1403$, $95 \%$ CI $=-1.5529$ to 5.2558 ) between the two groups. The incidence of mothers with two or more risk factors in neonates with biochemical evidence of sepsis was statistically significant when compared to the control group ( $p=<0.0001,95 \%$ $\mathrm{CI}=23.0629$ to 44.1866$)$. 


\section{Discussion}

In 1995 the UK incidence of early-onset sepsis in term neonates was 0.5 per 1,000 and the mortality rate was about $10 \%{ }^{8}$. The NICE clinical guideline 62 of 2008 reported the prevalence of early-onset GBS disease in England and Wales to be as 0.41.4 per 1000 live births (equivalent to about 340 babies per year) ${ }^{2}$. In Asia, in 2004, the reported incidence of neonatal sepsis varied from 7.1 to 38 per 1000 live births ${ }^{9}$. From 1995-96 a hospital in Vellore, India, had an incidence of early neonatal sepsis of $22.9 \%{ }^{10}$. In Africa, in 2004, the reported incidence of neonatal sepsis - early and late onsetvaried from 6.5 to 23 per 1000 live births. During the same period, in South America and the Caribbean, the incidence varied from 3.5 to 8.9 per 1000 live births ${ }^{9}$. In the Neonatal Care Unit, University Paediatric Unit, Colombo North Teaching Hospital, January to December 1996 the incidence of septicaemia - early and late onset was 24.4 per 1000 live births and case fatality rate was $11.2 \%{ }^{11}$.

Congenital pneumonia was responsible for 4.5 neonatal deaths per 100,000 births per year in the $\mathrm{UK}^{12}$. At Maulana Azad Medical College and Associated Lok Nayak Hospital, New Delhi, India, in 2001 pneumonia was found to be the most common cause $(62.8 \%)$ of respiratory distress in term neonates ${ }^{13}$. The incidence of bacterial meningitis in England and Wales from 1985-7 was 0.2 per 1000 live births. The mortality rate of GBS meningitis was $22 \%{ }^{14}$. In Christian Medical College Hospital, Vellore, Tamil Nadu, India the incidence of neonatal meningitis was 0.2 per 1000 live births in $1995-1996^{10}$.

Antenatal care (NICE clinical guideline 62, 2008) identified maternal intrapartum colonisation (or infection) with GBS as the leading cause of serious neonatal infection in the UK. The guideline noted that an estimated $28 \%$ of mothers in the UK carry GBS, (with no association with maternal age or parity) ${ }^{2}$. In the U.S.A. from 2002 to 2008 prolonged second stage was associated with neonatal morbidity including sepsis in nulliparous women with epidural: $2.6 \%$ versus $1.2 \%$ without epidural ${ }^{15}$. Estimates of the risks of early-onset GBS disease with PROM $>18$ hours is 1:476 according to Nottingham Neonatal Service ${ }^{16}$. Fifty six percent of neonates with early onset sepsis (EOS) in the NICU of a Paediatric Government Hospital of New Delhi, India had PROM more than 18 hours, $35.4 \%$ had more than 3 vaginal examinations ${ }^{17}$.

In one study in the United States neonatal pneumonia, sepsis and perinatal death in term infants did not occur in the absence of chorioamnionitis but occurred, respectively, in $4 \%$,
$8 \%$ and $2 \%$ of term deliveries associated with chorioamnionitis ${ }^{18}$. Twenty two percent of neonates with EOS in the NICU of a Paediatric Government Hospital of New Delhi, India had foul smelling/meconium stained liquor and $19.5 \%$ had maternal fever 2 weeks prior to delivery ${ }^{17}$. The incidence of early onset neonatal septicaemia in SJGH is more than that found in the U.K. but less than in India. The incidence of congenital pneumonia was less than what was found in India. The incidence of meningitis was higher than that found in U.K. and India. The number of mothers of term neonates with EOS having PROM for more than 18 hours in our study is more than in England but less than in other developing countries. More mothers had prolonged labour room stay than in developed countries. More mothers had 3 or more vaginal examinations than in India.

In our study, $1.8 \%$ of mother had foul smelling liquor as a risk factor, which was lower than in developing countries. Only $1.2 \%$ of mothers had high vaginal swab positive for GBS or a previous baby with GBS infection compared with $28 \%$ of mothers in the $\mathrm{UK}^{2}$. This is probably due to GBS screening being done regularly in the UK. Very few local studies have been carried out to estimate the incidence of EOS despite it being a common problem. None of the existing studies have compared incidence of sepsis with the incidence of maternal risk factors.

Unfortunately some babies who develop symptoms after discharge, at 24 to 72 hours of age may not get re-admitted to SJGH as the occurrence of this is beyond our control, this could not be assessed or avoided. A future study that may be helpful would be the effect of maternal risk factors in preterm neonates with sepsis.

\section{Conclusions}

In the current study there was a statistically significant increase in the incidence of PROM more than 18 hours, labour room stay more than 9 hours and 3 or more vaginal examinations in the neonates with biochemical evidence of sepsis. There was no significant difference in incidence of induction of labour, maternal fever, HVS positive for GBS and foul smelling liquor in neonates with biochemical evidence of sepsis.

\section{References}

1. Deorari A, editor. Neonatal sepsis. In Teaching Aids on Newborn Care [Monograph on the internet]. WHO-CC for Training \& Research in Newborn Care; 2005. Available from: http://www.newbornwhocc.org/pdf/teachi ng-aids/neonatalsepsis.pdf 
2. National Collaborating Centre for Women's and Children's Health (UK). NICE Clinical Guideline 149: Antibiotics for Early-Onset Neonatal Infection: Packham K, editor Antibiotics for the Prevention and Treatment of Early Onset Neonatal Infection. London (UK): RCOG Press; 2012 August. Available from: https://www.ncbi.nlm.nih.gov/pubmedhea 1th/PMH0051817/

3. Rajindrajith S, Mettananda S, Adihetti D, Goonawardana R, Devanarayana NM. Neonatal mortality in Sri Lanka: timing, causes and distribution. Journal of Maternal-Fetal and Neonatal Medicine 2009; 22(9): 791-6. https://doi.org/10.3109/147670509029945 49

PMid: 19526423

4. Sri Lanka College of Paediatricians. Weerasekera M, Rodrigo R, Lucas N, Rowel D editor. National guidelines for new-born care. Colombo (Sri Lanka): Ministry of Health; 2014.

5. National Collaborating Centre for Women's and Children's Health(UK). NICE Clinical Guideline 190: Intrapartum Care: Care of healthy women and their babies during childbirth; 2014 December. Available from: https://www.nice.org.uk/guidance/cg190

6. Anderson-Berry AL, Bellig LL, Bryan LO. Rosenkrantz $\mathrm{T}$ editor. Neonatal Sepsis Clinical Presentation [monograph on the Internet]. Medscape; 2015 Dec. Available from:

http://emedicine.medscape.com/article/97 8352-overview

7. Polin RA, the Committee on Fetus and Newborn. Management of Neonates with Suspected or Proven Early-Onset Bacterial Sepsis. Pediatrics 2012; 129(5): 1007-10. https://doi.org/10.1542/peds.2012-0541 PMid: 22547779

8. Du Pont-Thibodeau G, Joyal J-S, Lacroix $\mathrm{J}$. Management of neonatal sepsis in term newborns. F1000Prime Reports [seri Internet]. 2014 Aug [cited 2014 Aug 1];6(67). Available from https://www.ncbi.nlm.nih.gov/pmc/articles /PMC4126544/
9. Vergnano S, Sharland M, Kazembe $\mathrm{P}$, Mwansambo C, Heath P. Neonatal sepsis: an international perspective. Archives of Disease in Childhood Fetal and Neonatal Edition 2005; 90(3):122.

https://doi.org/10.1136/adc.2002.022863

PMid: 15846011 PMCid: PMC1721871

10. Kuruvilla KA, Pillai S, Jesudason M, Jana AK. Bacterial Profile of Sepsis in a Neonatal Unit in South India. Indian Pediatrics 1998; 35(9): 851-8.

PMid: 10216593

11. Karunasekera KA, Pathirana D. A preliminary study on neonatal septicaemia in a tertiary referral hospital paediatric unit. Ceylon Medical Journal 1999; 44(2): 81-86.

PMid: 10565074

12. Tambe P, Sammons HM, ChoonaraI I. Why do young children die in the UK? A comparison with Sweden. Archives of Disease in Childhood 2015 October; 100(10): 928-31.

https://doi.org/10.1136/archdischild-2014308059

PMid: 26272911 PMCid: PMC4602244

13. Mathur NB, Garg K, Kumar S. Respiratory distress in neonates with special reference to pneumonia. Indian Pediatrics 2002; 39(6): 529-37.

PMid: 12084946

14. Heath PT, Ifeanyichukwu OO, Oeser C. Neonatal meningitis:Can we do better?. Curtis N, Finn A, Pollard AJ, editors. Hot topics in infection and immunity in children. VIII Advances in Experimental Medicine and Biology. New York:Springer;2011. [p. 11-24].

15. Laughon SK, Berghella V, Reddy UM, Sundaram R, Lu Z, Hoffman MK. Neonatal and maternal outcomes with prolonged second stage of labor. Obstetrics and Gynecology 2014; 124(1): 57-67.

https://doi.org/10.1097/AOG.0000000000 000278

PMid: 24901265 PMCid: PMC4065200

16. Mackay K, Wardle S. Management of babies born to mothers with risk factors. Nottingham Neonatal Service - Clinical Guidelines [seri Internet]. Nottingham 2014 Feb; 7. 
17. Jajoo M, Kapoor K, Garg LK, Manchanda $\mathrm{V}$, Mittal SK. To study the incidence and risk factors of early onset neonatal sepsis in an out born neonatal intensive care unit of India. Journal of Clinical Neonatology 2015; 4(2): 91-95.

https://doi.org/10.4103/2249-4847.154106

18. Yoder PR, Gibbs RS, Blanco JD, Castaneda YS, St Clair PJ. A prospective, controlled study of maternal and perinatal outcome after intra-amniotic infection at term. American Journal of Obstretics and Gynecology 1983; 145(6): 695-701.

https://doi.org/10.1016/00029378(83)9057

$5-6$ 